\title{
Two-dimensional Poisson Trees converge to the Brownian web
}

\author{
P.A. Ferrari ${ }^{\mathrm{a}, *, 1}$, L.R.G. Fontes ${ }^{\mathrm{a}, 1}$, Xian-Yuan $\mathrm{Wu}^{\mathrm{b}, 2}$ \\ ${ }^{a}$ Instituto de Matemática e Estatística, Universidade de São Paulo, Rua do Matão 1010, 05508-090 São Paulo - SP, Brazil \\ ${ }^{\mathrm{b}}$ Department of Mathematics, Capital Normal University, Beijing 100037, China
}

Received 20 October 2003; received in revised form 6 April 2004; accepted 8 June 2004

Available online 5 February 2005

\begin{abstract}
The Brownian web can be roughly described as a family of coalescing one-dimensional Brownian motions starting at all times in $\mathbb{R}$ and at all points of $\mathbb{R}$. The two-dimensional Poisson tree is a family of continuous time one-dimensional random walks with uniform jumps in a bounded interval. The walks start at the space-time points of a homogeneous Poisson process in $\mathbb{R}^{2}$ and are in fact constructed as a function of the point process. This tree was introduced by Ferrari, Landim and Thorisson. By verifying criteria derived by Fontes, Isopi, Newman and Ravishankar, we show that, when properly rescaled, and under the topology introduced by those authors, Poisson trees converge weakly to the Brownian web.
\end{abstract}

() 2005 Elsevier SAS. All rights reserved.

\section{Résumé}

La «toile brownienne» peut approximativement être décrite comme une famille coalescente de mouvements browniens unidimensionnels commençant, en tout temps de la droite réelle, à partir de tout point de la droite réelle. On montre qu'elle peut être approchée en un sens faible par une famille d'arbres poissonniens bidimensionnels.

(c) 2005 Elsevier SAS. All rights reserved.

MSC: $60 \mathrm{~K} 35 ; 60 \mathrm{~F} 17$

Keywords: Brownian web; Poisson tree; Coalescing one-dimensional Brownian motions

\footnotetext{
* Corresponding author.

E-mail addresses: pablo@ime.usp.br (P.A. Ferrari), lrenato@ime.usp.br (L.R.G. Fontes), wuxy@mail.cnu.edu.cn, xianyuan_wu@hotmail.com (X.-Y.Wu).

1 Partially supported by CNPq grants 520811/96-8, 300576/92-7 and 662177/96-7 (PRONEX) and FAPESP grant 99/11962-9.

2 Partially supported by FAPESP grant 01/02577-6, NSFC grant 10301023 and Foundation of Beijing Education Bureau.
} 


\section{Introduction and results}

Let $S$ be a two-dimensional homogeneous Poisson process of parameter $\lambda . S$ is a random subset of $\mathbb{R}^{2}, s \in S$ has coordinates $s_{1}, s_{2}$.

For $x=\left(x_{1}, x_{2}\right) \in \mathbb{R}^{2}, t \geqslant x_{2}$ and $r>0$, let $M(x, t, r)$ be the following rectangle

$$
M(x, t, r):=\left\{\left(x_{1}^{\prime}, x_{2}^{\prime}\right):\left|x_{1}^{\prime}-x_{1}\right| \leqslant r, x_{2} \leqslant x_{2}^{\prime} \leqslant t\right\} .
$$

As $t$ grows, the rectangle gets longer. The first time $t$ that $M(x, t, r)$ hits some (or another, when $x \in S$ ) point of $S$ is called $\tau(x, S, r)$; this is defined by

$$
\tau(x, S, r):=\inf \left\{t>x_{2}: M(x, t, r) \cap(S \backslash\{x\}) \neq \emptyset\right\} .
$$

The hitting point is the point $\alpha(x) \in S$ defined by

$$
\alpha(x):=M(x, \tau(x, S, r), r) \cap(S \backslash\{x\}),
$$

which consists of a unique point almost surely. If $x=$ some $s \in S$, we say that $\alpha(x)=\alpha(s)$ is the mother of $s$ and that $s$ is a daughter of $\alpha(s)$. Let $\alpha^{0}(x)=x$ and iteratively, for $n \geqslant 1, \alpha^{n}(x)=\alpha\left(\alpha^{n-1}(x)\right)$. For the case of $x=$ some $s \in S, \alpha^{n}(x)=\alpha^{n}(s)$ is the $n$th grand mother of $s$.

Now let $G=(V, E)$ be the random directed graph with vertices $V=S$ and edges $E=\{(s, \alpha(s)): s \in S\}$. Ferrari, Landim and Thorisson [4] proved that $G$ is a tree with a unique connected component and called it the two-dimensional Poisson tree. The drainage networks of Gangopadhyay, Roy and Sarkar [9] can be viewed as a discrete space, long range version of the Poisson tree.

The Poisson tree induces sets of continuous paths. For any $s=\left(s_{1}, s_{2}\right) \in S$, define the path $X^{s}$ in $\mathbb{R}^{2}$ as the linearly interpolated line composed by all edges $\left\{\left(\alpha^{n-1}(s), \alpha^{n}(s)\right): n \in \mathbb{N}\right\}$ of $G$. Let

$$
X:=\left\{X^{s}: s \in S\right\},
$$

which we also call the Poisson web.

Clearly $X$ depends on $\lambda>0$ and $r>0$; if necessary we denote it by $X(\lambda, r)$. Take $\lambda=\lambda_{0}=\sqrt{3} / 6, r=r_{0}=\sqrt{3}$, and let

$$
X_{1}:=X\left(\lambda_{0}, r_{0}\right) ; \quad X_{\delta}:=\left\{\left(\delta x_{1}, \delta^{2} x_{2}\right) \in \mathbb{R}^{2}:\left(x_{1}, x_{2}\right) \in X_{1}\right\}, \quad \delta \in(0,1] .
$$

Namely, $X_{\delta}$ is the diffusive rescaling of $X_{1}$.

Our main result is a proof that $X_{\delta}$ converges in distribution to the Brownian web characterized in [7]. [7] introduces a metric space $(\Pi, d)$ of continuous paths firstly, and then defines the Hausdorff metric space $\left(\mathcal{H}, d_{\mathcal{H}}\right)$ of compact subsets of $(\Pi, d)$, where $d_{\mathcal{H}}$ and $d$ are the corresponding metric functions. Denote by $\mathcal{F}_{\mathcal{H}}$ the corresponding Borel $\sigma$-algebra generated by $d_{\mathcal{H}}$. The Brownian web is characterized there as a $(\mathcal{H}, \mathcal{F} \mathcal{H})$-valued random variable $\overline{\mathcal{W}}$ (or its distribution $\mu_{\overline{\mathcal{W}}}$ ) whose "finite-dimensional distributions" (in a sense made precise in [7]) are coalescing one-dimensional Brownian motions.

Theorem 1.1. The rescaled Poisson trees $X_{\delta}$ converge in distribution to the standard Brownian web as $\delta \rightarrow 0$.

Given $t_{0} \in \mathbb{R}, t>0, a<b$, and a $\left(\mathcal{H}, \mathcal{F}_{\mathcal{H}}\right)$-valued random variable $V$, let $\eta_{V}\left(t_{0}, t ; a, b\right)$ be the $\{0,1,2, \ldots, \infty\}$ valued random variable giving the number of distinct points in $\mathbb{R} \times\left\{t_{0}+t\right\}$ that are touched by paths in $V$ which also touch some point in $[a, b] \times\left\{t_{0}\right\}$. By the weak convergence criteria given in [7], for any $\left(\mathcal{H}, \mathcal{F}_{\mathcal{H}}\right)$-valued random variables $\left\{X_{n}\right\}_{n=1}^{\infty}$ with noncrossing paths, to prove that $X_{n}$ converges to the standard Brownian web, one may verify the following: For some countable dense set $\mathcal{D}$ in $\mathbb{R}^{2}$,

$\left(\mathrm{I}_{1}\right)$ There exist $\theta_{n}^{y} \in X_{n}$ such that for any deterministic $y_{1}, \ldots, y_{m} \in \mathcal{D}, \theta_{n}^{y_{1}}, \ldots, \theta_{n}^{y_{m}}$ converge in distribution as $n \rightarrow \infty$ to coalescing Brownian motions (with unit diffusion constant) starting at $y_{1}, \ldots, y_{m}$; 
$\left(\mathrm{B}_{1}\right) \lim \sup _{n \rightarrow \infty} \sup _{\left(a, t_{0}\right) \in \mathbb{R}^{2}} \mathbb{P}\left(\eta_{X_{n}}\left(t_{0}, t ; a, a+\epsilon\right) \geqslant 2\right) \rightarrow 0$ as $\epsilon \rightarrow 0+$;

$\left(\mathrm{B}_{2}\right) \epsilon^{-1} \lim \sup _{n \rightarrow \infty} \sup _{\left(a, t_{0}\right) \in \mathbb{R}^{2}} \mathbb{P}\left(\eta_{X_{n}}\left(t_{0}, t ; a, a+\epsilon\right) \geqslant 3\right) \rightarrow 0$ as $\epsilon \rightarrow 0+$.

To prove the main result, we show in Section 2 that the Poisson webs $X_{\delta}$ satisfy the three hypotheses above. The verification of $\mathrm{I}_{1}$, on Subsection 2.1, relies on a comparison with independent paths and on the almost sure coalescence of the Poisson web paths with each other. See Lemma 2.3.

In Subsection 2.2, an FKG inequality enjoyed by the distribution of a single Poisson web path (Lemma 2.6) and the $\mathrm{O}\left(t^{-1 / 2}\right)$ decay of the coalescence time of two such paths (Lemma 2.7), combined with $\mathrm{I}_{1}$, yield both $\mathrm{B}_{1}$ and $\mathrm{B}_{2}$. The argument is similar in spirit to the one for establishing weak convergence of coalescing random walks to the Brownian web in [7]. The details are nonetheless substantially different, more involved here, due to the dependence between the paths of the Poisson tree before coalescence. See the remark at the end of the paper. See also [3] for more details.

Working out a second example of a process in the basin of attraction of the Brownian web (the first example is ordinary one-dimensional coalescing random walks) that is natural on one side, and that requires substantial technical attention on another side, is the primary point of this paper. Its main result may have an applied interest, e.g. in the context of drainage networks. The convergence results here may lead to rigorous/alternative verification of some of the scaling theory for those networks. See [13]. Ordinary one-dimensional coalescing random walks starting from all space-time points have also been proposed as model of a drainage network [14], so the latter remark applies to them as well. Another application would be in obtaining aging results from the scaling limit results for systems that could be modeled by Poisson webs, like drainage networks. For the relation between aging and scaling limits, see e.g. [7,5,6,8], and references therein.

\section{Proofs}

Coalescing random walks. Let $S$ be the Poisson process with parameter $\lambda>0$, fix some $r>0$. For any $x=$ $\left(x_{1}, x_{2}\right) \in \mathbb{R}^{2}$, let $\tau^{n}(x)=\left[\alpha^{n}(x)\right]_{2}, n \geqslant 0$, be the second coordinate of $\alpha^{n}(x)$ and consider $\left\{\xi^{x}(t): t \geqslant x_{2}\right\}$ as the continuous time Markov process defined by

$$
\xi^{x}(t)=\left[\alpha^{n}(x)\right]_{1}, \text { the first coordinate of } \alpha^{n}(x) ; \quad t \in\left[\tau^{n}(x), \tau^{n+1}(x)\right), \quad n \geqslant 0 .
$$

We remark that for any fixed $\left\{x^{i}\right\}_{i=1}^{m}$, with $x^{i}=\left(x_{1}^{i}, x_{2}^{i}\right) \in \mathbb{R}^{2}$ for $i=1, \ldots, m,\left\{\left(\xi^{x^{i}}(t): t \geqslant x_{2}^{i}\right), i=1, \ldots, m\right\}$ defines a finite system of coalescing random walks starting at the space-time points $x^{1}, \ldots, x^{m}$.

For $x=\left(x_{1}, x_{2}\right) \in \mathbb{R}^{2}$, let $x_{\delta}=\left(\delta^{-1} x_{1}, \delta^{-2} x_{2}\right), \delta \in(0,1]$. For the single random walk starting at $x=\left(x_{1}, x_{2}\right)$, $\xi^{x}(\cdot)$, defined in the last paragraph, the diffusive rescaling is

$$
\xi_{\delta}^{x}(t):=\delta \xi^{x_{\delta}}\left(\delta^{-2} t\right), \quad \text { for } t \geqslant x_{2} ; \delta \in(0,1] .
$$

Since the characterizing theorem and the weak convergence criteria given in [7] apply to continuous paths only, we need to replace the original processes by their linearly interpolated versions:

$$
\bar{\xi}_{\delta}^{x}(t)=\delta\left\{\xi^{x_{\delta}}\left(\tau^{n}\left(x_{\delta}\right)\right)+\frac{\delta^{-2} t-\tau^{n}\left(x_{\delta}\right)}{\tau^{n+1}\left(x_{\delta}\right)-\tau^{n}\left(x_{\delta}\right)}\left(\xi^{x_{\delta}}\left(\tau^{n+1}\left(x_{\delta}\right)\right)-\xi^{x_{\delta}}\left(\tau^{n}\left(x_{\delta}\right)\right)\right)\right\},
$$

for $t \geqslant x_{2}$ such that $\delta^{-2} t \in\left[\tau^{n}\left(x_{\delta}\right), \tau^{n+1}\left(x_{\delta}\right)\right), n \geqslant 0 ; \delta \in(0,1], x \in \mathbb{R}^{2}$. Denote by $\bar{\xi}_{\delta}^{x}$ the corresponding continuous path in $\mathbb{R}^{2}$ and note that $\bar{\xi}_{1}^{s}$ is just $X^{s}$ in (1.4) with $s \in S$. It is straightforward to see that $\bar{\xi}_{\delta}^{x} \in X_{\delta}$, the Poisson web defined by (1.5), if and only if $x_{\delta} \in S$.

Let

$$
\theta_{\delta}^{x}:= \begin{cases}\bar{\xi}_{\delta}^{x} & \text { if } x_{\delta} \in S \\ \bar{\xi}_{\delta}^{\left(\delta\left[\alpha\left(x_{\delta}\right)\right]_{1}, \delta^{2}\left[\alpha\left(x_{\delta}\right)\right]_{2}\right)} & \text { otherwise }\end{cases}
$$


In this way, for all $x \in \mathbb{R}^{2}$ and $\delta \in(0,1], \theta_{\delta}^{x} \in X_{\delta}$. Note that the paths defined by (2.3) and (2.4) depend on the choice of $\lambda>0$ and $r>0$. In case of necessity, we denote them by $\bar{\xi}_{\delta}^{x}(\lambda, r)$ and $\theta_{\delta}^{x}(\lambda, r)$.

The following is an application of the classical Donsker's theorem [2] to our case.

Lemma 2.1. If $\lambda=\lambda_{0}=\sqrt{3} / 6, r=r_{0}=\sqrt{3}$, then $\bar{\xi}_{\delta}^{x}$ converges in distribution as $\delta \rightarrow 0$ to $B^{x}$, the Brownian path with unit diffusion coefficient starting from space-time point $x=\left(x_{1}, x_{2}\right) \in \mathbb{R}^{2}$.

For any $x^{1}, \ldots, x^{m} \in \mathbb{R}^{2}, m \in \mathbb{N}$, regard $\left(\bar{\xi}_{\delta}^{x^{1}}, \ldots, \bar{\xi}_{\delta}^{x^{m}}\right)$ and $\left(\theta_{\delta}^{x^{1}}, \ldots, \theta_{\delta}^{x^{m}}\right)$ as random variables in the product metric space $\left(\Pi^{m}, d^{* m}\right)$, where $d^{* m}$ is a metric on $\Pi^{m}$ such that the topology generated by it coincides with the corresponding product topology. Here we choose and define

$$
d^{* m}\left[\left(\xi^{1}, \ldots, \xi^{m}\right),\left(\zeta^{1}, \ldots, \zeta^{m}\right)\right]=\max _{1 \leqslant i \leqslant m} d\left(\xi^{i}, \zeta^{i}\right)
$$

for all $\left(\xi^{1}, \ldots, \xi^{m}\right),\left(\zeta^{1}, \ldots, \zeta^{m}\right) \in \Pi^{m}$, where $d$ was defined in [7]. The next result follows immediately from the definition.

\section{Lemma 2.2.}

$$
\mathbb{P}_{\lambda}\left\{d^{* m}\left[\left(\bar{\xi}_{\delta}^{x^{1}}, \ldots, \bar{\xi}_{\delta}^{x^{m}}\right),\left(\theta_{\delta}^{x^{1}}, \ldots, \theta_{\delta}^{x^{m}}\right)\right] \geqslant \epsilon\right\} \rightarrow 0, \quad \text { as } \delta \rightarrow 0
$$

for all $\epsilon>0, \lambda>0, r>0$, and $x^{1}, \ldots, x^{m} \in \mathbb{R}^{2}, m \in \mathbb{N}$, where $\mathbb{P}_{\lambda}$ is the probability distribution of $S$, the Poisson process with parameter $\lambda$.

\subsection{Convergence in finite-dimensional cases: verification of condition $I_{1}$}

Let $\mathcal{D}$ be a countable dense set of points in $\mathbb{R}^{2}$, to verify condition $\mathrm{I}_{1}$, by Lemma 2.2 , we only need to prove the following.

Lemma 2.3. $\left(\bar{\xi}_{\delta}^{y^{1}}, \ldots, \bar{\xi}_{\delta}^{y^{m}}\right)$ converges in distribution as $\delta \rightarrow 0$ to coalescing Brownian motions (with unit diffusion constant) starting at $y^{1}, \ldots, y^{m}(\in \mathcal{D})$.

For the finite system of coalescing random walks defined in the last subsection, Ferrari, Landim and Thorisson [4] proved that, for any $x, y \in \mathbb{R}^{2}$, the random walks $\xi^{x}(t)$ and $\xi^{y}(t), t \geqslant x_{2} \vee y_{2}$ will meet and then coalesce almost surely. This also follows from Lemma 2.7 below. The following is a corollary of this result.

Lemma 2.4. For any $\lambda>0, r>0, \mathbb{P}_{\lambda}\left\{\sup _{t \geqslant x_{2}}\left|\bar{\xi}_{1}^{x}(t)-\bar{\xi}_{1}^{y}(t)\right| \geqslant \sigma\right\} \rightarrow 0$, as $\sigma \rightarrow \infty$ for all $x, y \in \mathbb{R}^{2}$ with $x_{2}=y_{2}$.

Now, for any $m$ distinct points $y^{1}, \ldots, y^{m} \in \mathcal{D}$ and $\delta \in(0,1]$. Let $\bar{\xi}_{\delta}^{y^{1}}, \ldots, \bar{\xi}_{\delta}^{y^{m}}$ be the $m$ rescaled continuous random paths defined in (2.3) from the same Poisson process with $\lambda=\lambda_{0}=\sqrt{3} / 6$ and $r=r_{0}=\sqrt{3}$. Having $\left(\bar{\xi}_{\delta}^{y^{1}}, \ldots, \bar{\xi}_{\delta}^{y^{m}}\right)$ as a random element in $\Pi^{m}$, we want to define a function $f_{\delta}$ from it to $\Pi^{m}$. This is our main idea for the verification of condition $\mathrm{I}_{1}$ : we define what we call " $\delta$-coalescence" of the random paths $\bar{\xi}_{\delta}^{y^{1}}, \ldots, \bar{\xi}_{\delta}^{y^{m}}$ in such a way that, in the system $f_{\delta}\left(\bar{\xi}_{\delta}^{y^{1}}, \ldots, \bar{\xi}_{\delta}^{y^{m}}\right)$, before any $\delta$-coalescence, the paths involved are independent.

We define $f_{\delta}$ by renewing the whole system step by step as follows. Consider $\left(\xi_{\delta}^{y^{1}}, \ldots, \xi_{\delta}^{y^{m}}\right)$, the rescaled finite system of coalescing random walks starting at the space-time points $y^{1}, \ldots, y^{m}$. Let $\gamma_{\delta, 0}=\min \left(y_{2}^{1}, \ldots, y_{2}^{m}\right)$, and we assume that $\delta>0$ is close enough to 0 , so that, in particular, the following stopping times we need are well defined. 
For $\left(\xi_{\delta}^{y^{1}}, \ldots, \xi_{\delta}^{y^{m}}\right)$, as $t\left(>\gamma_{\delta, 0}\right)$ grows, let $\gamma_{\delta, k}, 1 \leqslant k \leqslant m-1$, be the time when the $k$ th $\delta$-coalescence occurs. We say a $\delta$-coalescence occurs at time $t_{0}$, if $t_{0}$ is the time when two particles get within a distance smaller than $2 \sqrt{3} \delta$. Once a $\delta$-coalescence occurs, we renew the system by coalescing the two particles to be the left one and then wait for the next $\delta$-coalescence.

Denote the linearly interpolated versions of the resulting object after renewing $m-1$ times by $f_{\delta}\left(\bar{\xi}_{\delta}^{y^{1}}, \ldots, \bar{\xi}_{\delta}^{y^{m}}\right)$ and, with that, finish the definition of the function $f_{\delta}$. Clearly, for $\delta \in(0,1]$ small enough, we have

$$
-\infty<\gamma_{\delta, 0}<\gamma_{\delta, 1}<\cdots<\gamma_{\delta, m-1}<\infty
$$

and the function $f_{\delta}$ is well defined almost surely.

Now, suppose that $\tilde{\xi}_{\delta}^{y^{i}}$ has the same distribution as $\bar{\xi}^{y^{i}}, 1 \leqslant i \leqslant m$, and, as a random element in $\Pi^{m}$, $\left(\tilde{\xi}_{\delta}^{y^{1}}, \ldots, \tilde{\xi}_{\delta}^{y^{m}}\right)$ has independent components. It is easy to see that the function $f_{\delta}$ is also well defined for the random paths $\left(\tilde{\xi}_{\delta}^{y^{1}}, \ldots, \tilde{\xi}_{\delta}^{y^{m}}\right)$. Let $C_{\delta} \subset \Pi^{m}$ be such that

$$
\mathbb{P}\left\{\left(\tilde{\xi}_{\delta}^{y^{1}}, \ldots, \tilde{\xi}_{\delta}^{y^{m}}\right) \in C_{\delta}\right\}=1
$$

and, on $C_{\delta}, f_{\delta}$ is well defined.

For any $y^{1}, \ldots, y^{m} \in \mathcal{D}$, let $B^{y^{1}}, \ldots, B^{y^{m}}$ be $m$ independent Brownian paths starting at space-time points $y^{1}, \ldots, y^{m}$, respectively. As Arratia did in [1], we construct a set $\left\{\tilde{B}^{y^{1}}, \ldots, \tilde{B}^{y^{m}}\right\}$ of $m$ one-dimensional coalescing Brownian motions starting at $y^{1}, \ldots, y^{m}$ by defining an almost surely continuous function $f$ from $\Pi^{m}$ to $\Pi^{m}$ as follows. The first Brownian path of the set, $\tilde{B}^{y^{1}}$, is $B^{y^{1}}$ itself. Once we have $\left\{\tilde{B}^{y^{1}}, \ldots, \tilde{B}^{y^{k-1}}\right\}$, we define $\tilde{B}^{y^{k}}$ to be equal to $B^{y^{k}}$ till that path first hits any of $\tilde{B}^{y^{1}}, \ldots, \tilde{B}^{y^{k-1}}$, say $\tilde{B}^{\hat{y}}$; thence on it coincides with $\tilde{B}^{\hat{y}}$. This procedure is a.s. well defined, and the system resulting after $m-1$ steps is the so-called one-dimensional coalescing Brownian motions starting at space-time points $y^{1}, \ldots, y^{m}$, which we denote by $f\left(B^{y^{1}}, \ldots, B^{y^{m}}\right)$, as a function of the $m$ independent Brownian motions $B^{y^{1}}, \ldots, B^{y^{m}}$.

Lemma 2.5. Let $\left(\bar{\xi}_{\delta}^{y^{1}}, \ldots, \bar{\xi}_{\delta}^{y^{m}}\right)$ be the $m$ rescaled continuous random paths defined in (2.3) from the same Poisson process with $\lambda=\lambda_{0}=\sqrt{3} / 6$ and $r=r_{0}=\sqrt{3}$, and $\left(\tilde{\xi}_{\delta}^{y^{1}}, \ldots, \tilde{\xi}_{\delta}^{y^{m}}\right)$ have independent components and $\tilde{\xi}_{\delta}^{y^{i}}$ have the same distribution as $\bar{\xi}^{y^{i}}$ for all $1 \leqslant i \leqslant m$. Then,

(a) $f_{\delta}\left(\bar{\xi}_{\delta}^{y^{1}}, \ldots, \bar{\xi}_{\delta}^{y^{m}}\right)$ has the same distribution as $f_{\delta}\left(\tilde{\xi}_{\delta}^{y^{1}}, \ldots, \tilde{\xi}_{\delta}^{y^{m}}\right)$;

(b) $f_{\delta}\left(\tilde{\xi}_{\delta}^{y^{1}}, \ldots, \tilde{\xi}_{\delta}^{y^{m}}\right)$ converges in distribution to $f\left(B^{y^{1}}, \ldots, B^{y^{m}}\right)$ as $\delta \rightarrow 0$;

(c) for any $\epsilon>0, \mathbb{P}\left\{d^{* m}\left[f_{\delta}\left(\bar{\xi}_{\delta}^{y^{1}}, \ldots, \bar{\xi}_{\delta}^{y^{m}}\right),\left(\bar{\xi}_{\delta}^{y^{1}}, \ldots, \bar{\xi}_{\delta}^{y^{m}}\right)\right] \geqslant \epsilon\right\} \rightarrow 0$, as $\delta \rightarrow 0$. $d^{* m}$ was defined in (2.5).

Proof. (a), (c) Immediate from the definition of $f_{\delta}$ and Lemma 2.4. For (b), it is straightforward to check that, for any $c=\left(c^{1}, \ldots, c^{m}\right) \in C, c_{\delta}=\left(c_{\delta}^{1}, \ldots, c_{\delta}^{m}\right) \in C_{\delta}, d^{* m}\left(c_{\delta}, c\right) \rightarrow 0$ implies $d^{* m}\left(f_{\delta}\left(c_{\delta}\right), f(c)\right) \rightarrow 0$ as $\delta \rightarrow 0$. Thus, an extended continuous mapping theorem of Mann and Wald [11], Prohorov [12] (see also Theorem 3.27 of [10]) gives (b).

Lemma 2.3 is an immediate consequence of Lemma 2.5. Thus, condition $\mathrm{I}_{1}$ for the Poisson web $X_{\delta}, \delta \in(0,1]$ follows from Lemma 2.2 .

\subsection{Verification of conditions $B_{1}$ and $B_{2}$}

Consider the Poisson process $S$ with parameter $\lambda>0$ and the corresponding Poisson tree $X:=X(\lambda, r)$ defined in (1.4) with respect to some fixed $r>0$. Given $t_{0} \in \mathbb{R}, t>0, a, b \in \mathbb{R}$ with $a<b$, let $\eta_{X}\left(t_{0}, t ; a, b\right)$ be the 
$\{0,1,2, \ldots, \infty\}$-valued random variable defined right after the statement of Theorem 1.1. Let $\bar{\eta}_{X}\left(t_{0}, t ; a, b\right)$ be another $\{0,1,2, \ldots, \infty\}$-valued random variable defined as the number of distinct points $y=\left(y_{1}, y_{2}\right) \in \mathbb{R} \times\left\{t_{0}+t\right\}$ such that there exists $s \in S$ with $s_{2} \leqslant t_{0}, \xi^{s}\left(t_{0}\right) \in[a, b]$ and $\xi^{s}\left(t_{0}+t\right)=\xi^{s}\left(y_{2}\right)=y_{1}$, where $\xi^{s}$ is the Markov process defined in (2.1). It is straightforward to see that, for any fixed $n \in \mathbb{N}$,

$$
\bar{\eta}_{X}\left(t_{0}, t ; a, b\right) \geqslant n \Rightarrow \eta_{X}\left(t_{0}, t ; a-2 r, b+2 r\right) \geqslant n \Rightarrow \bar{\eta}_{X}\left(t_{0}, t ; a-4 r, b+4 r\right) \geqslant n .
$$

This implies that, to verify conditions $\mathrm{B}_{1}, \mathrm{~B}_{2}$ for the Poisson trees $X_{\delta}$, we only need to verify the following $\mathrm{B}_{1}^{\prime}$ and $\mathrm{B}_{2}^{\prime}$.

$\left(\mathrm{B}_{1}^{\prime}\right) \lim \sup _{n \rightarrow \infty} \mathbb{P}\left(\bar{\eta}_{\delta_{n}}(0, t ; 0, \epsilon) \geqslant 2\right) \rightarrow 0$ as $\epsilon \rightarrow 0+$;

$\left(\mathrm{B}_{2}^{\prime}\right) \epsilon^{-1} \lim \sup _{n \rightarrow \infty} \mathbb{P}\left(\bar{\eta}_{\delta_{n}}(0, t ; 0, \epsilon) \geqslant 3\right) \rightarrow 0$ as $\epsilon \rightarrow 0+$

for any sequence of positive numbers $\left(\delta_{n}\right)$ such that $\lim _{n \rightarrow \infty} \delta_{n}=0$, where $\bar{\eta}_{\delta}=\bar{\eta}_{X_{\delta}}$, and we have used the space homogeneity of the Poisson point process to eliminate the $\sup _{\left(a, t_{0}\right) \in \mathbb{R}^{2}}$ and put $a=t_{0}=0$.

Here, we firstly introduce an FKG inequality for probability measures on the path space, which will play an important role in our proofs. Let $\xi=\xi^{(0,0)}$ be the random path starting at the origin defined in $(2.1)$; denote by $\bar{\Pi}$ the space of paths where $\xi$ takes value. We define a partial order “ $\preceq$ ” on $\bar{\Pi}$ as follows. Given $\pi_{1}, \pi_{2} \in \bar{\Pi}$,

$$
\pi_{1} \preceq \pi_{2} \text { if and only if } \pi_{1}(t)-\pi_{1}(s) \leqslant \pi_{2}(t)-\pi_{2}(s) \text { for all } t \geqslant s \geqslant 0 .
$$

Define increasing events in $\bar{\Pi}$ as usual. Denote by $\mu_{\xi}$ the distribution of $\xi$ on $\bar{\Pi}$.

Lemma 2.6 (FKG Inequality). $\mu_{\xi}$ satisfies the FKG inequality, namely, for any increasing events $A, B \subseteq \bar{\Pi}$, $\mu_{\xi}(A B) \geqslant \mu_{\xi}(A) \mu_{\xi}(B)$.

Proof. Follows by discretizing $\xi$ as a discrete time random walk, and then using FKG for its i.i.d. increments. Details can be found in [3].

Let $\xi^{(0,0)}, \xi^{(\gamma, 0)}, \gamma \geqslant r$, be two of the random walks defined in (2.1). Denote by $\Delta_{\gamma}$ the difference between them. Then, $\Delta_{\gamma}$ is a (space inhomogeneous) jump process in $[0, \infty)$ with absorbing state $0: \operatorname{In} x \in[r, \infty)$, it has rates $(2 r+x \wedge(2 r)) \lambda$ and jump laws

$$
v_{x}:=\frac{2 r-x \wedge(2 r)}{2 r+x \wedge(2 r)} \delta_{\{-x\}}+\frac{2(x \wedge(2 r))}{2 r+x \wedge(2 r)} U[r-x \wedge(2 r), r],
$$

where $\delta_{\{-x\}}$ is the usual Dirac measure and $U[r-x, r]$ is the uniform distribution on $[r-x, r]$. Let $\mathcal{T}=\inf \{t>0$ : $\left.\Delta_{2 r}(t)=\xi^{(0,0)}(t)-\xi^{(2 r, 0)}(t)=0\right\}$.

Lemma 2.7. There exists a constant $c>0$ such that $\mathbb{P}(\mathcal{T}>t) \leqslant c / \sqrt{t}$ for any $t>0$, where $c$ depends on $r$ and $\lambda$ only.

Proof. Follows by a coupling of $\Delta_{2 r}$ and a space homogeneous process which has the same transition distribution as $\Delta_{2 r}$ outside a neighborhood of the origin. Inside that neighborhood they are not too different, so that the result for $\Delta_{2}$ follows from that the analogous one for the homogeneous process, which is a random walk, and for which that result follows from standard arguments. Details can be found in [3].

Now, we begin to verify conditions $\mathrm{B}_{1}^{\prime}$ and $\mathrm{B}_{2}^{\prime}$. By Lemma 2.3, it is straightforward to get that,

$$
\limsup _{n \rightarrow \infty} \mathbb{P}\left(\eta_{\delta_{n}}(0, t ; 0, \epsilon) \geqslant 2\right)=2 \phi(\epsilon / \sqrt{2 t})-1,
$$


where $\delta_{n}$ is any sequence of positive numbers converging to 0 as $n \rightarrow \infty, \eta_{\delta}=\eta_{X_{\delta}}$, and $\phi(x)$ is the standard normal distribution function. By (2.9), (2.12) with $\bar{\eta}_{X_{\delta}}$ replacing $\eta_{X_{\delta}}$ also holds. This gives $\mathrm{B}_{1}^{\prime}$.

Verifying $\mathrm{B}_{2}^{\prime}$ for the Poisson web $X_{\delta}$ is equivalent to checking that for any $t>0$

$$
\epsilon^{-1} \limsup _{N \rightarrow \infty} \mathbb{P}\left(\bar{\eta}_{X_{1}}(0, t N ; 0, \epsilon \sqrt{N}) \geqslant 3\right) \rightarrow 0 \quad \text { as } \epsilon \rightarrow 0+,
$$

where $X_{1}$ is defined in (1.5).

For that, fix $t>0$. On the Poisson field $S$ with parameter $\lambda=\lambda_{0}=\sqrt{3} / 6$, choose $r=r_{0}=\sqrt{3}$, and then define $X_{1}$ as in (1.5). We first condition the probability in (2.13) on the set of points of intersection, in increasing order, of the paths $\xi^{s}, s \in S$, with $[0, \epsilon \sqrt{N}]$, denoted $\left\{K_{1}, \ldots, K_{J}\right\}$, where $J, K_{1}, \ldots, K_{J}$ are random variables, with $J$ an integer which can equal 0 (in which case set of intersection points is empty by convention). We note that by the definition of $\xi^{s}, s \in S$, no two distinct $K_{i}$ 's can be at distance smaller than $r_{0}$. For $\left\{x_{1}, \ldots, x_{n}\right\} \subset[0, \epsilon \sqrt{N}]$, let $\xi_{j}:=\xi^{\left(x_{j}, 0\right)}, 1 \leqslant j \leqslant n$, as in $(2.1)$. Let $\eta^{\prime}=\eta^{\prime}\left(x_{1}, \ldots, x_{n}\right)=\left|\left\{\xi_{j}(t N): 1 \leqslant j \leqslant n\right\}\right|$ (conventioned to be 0 if $\left.\left\{x_{1}, \ldots, x_{n}\right\}=\emptyset\right)$. Clearly, $J, K_{1}, \ldots, K_{J}$ depend only on the points of $S$ below time 0 . Thus, since $\eta^{\prime}$ depends only on the points of $S$ above and at time 0 for all $\left\{x_{1}, \ldots, x_{n}\right\} \subset[0, \epsilon \sqrt{N}]$, given $J=n, K_{1}=x_{1}, \ldots, K_{J}=x_{n}$, the probability in (2.13) equals $\mathbb{P}\left(\eta^{\prime} \geqslant 3\right)$.

We derive next an upper bound for the latter probability which is independent of $\left\{x_{1}, \ldots, x_{n}\right\}$. First, we enlarge, if necessary, the set $\left\{x_{1}, \ldots, x_{n}\right\}$ to make sure that $x_{1}=0, x_{n}=\epsilon \sqrt{N}$, and $r_{0} \leqslant x_{j}-x_{j-1} \leqslant 2 r_{0}$. This also ensures that $n \leqslant \epsilon \sqrt{N} / r_{0}+1$, and the enlargement can only increase the probability to be estimated. If $\eta^{\prime} \geqslant 3$, then there should be some $1 \leqslant j \leqslant n-1$ such that $\xi_{j-1}(t N)<\xi_{j}(t N)<\xi_{n}(t N)$. Hence,

$$
\begin{aligned}
\mathbb{P}\left(\eta^{\prime} \geqslant 3\right) & \leqslant \sum_{j=2}^{n-1} \mathbb{P}\left(\xi_{j-1}(t N)<\xi_{j}(t N)<\xi_{n}(t N)\right) \\
& =\sum_{j=2}^{n-1} \int_{\bar{\Pi}_{j}} \mathbb{P}\left(\xi_{j-1}(t N)<\xi_{j}(t N)<\xi_{n}(t N) \mid \xi_{j}=\pi\right) \mu_{\xi_{j}}(\mathrm{~d} \pi) \\
& =\sum_{j=2}^{n-1} \int_{\bar{\Pi}_{j}} \mathbb{P}\left(\xi_{j-1}(t N)<\xi_{j}(t N) \mid \xi_{j}=\pi\right) \mathbb{P}\left(\xi_{j}(t N)<\xi_{n}(t N) \mid \xi_{j}=\pi\right) \mu_{\xi_{j}}(\mathrm{~d} \pi)
\end{aligned}
$$

where $\bar{\Pi}_{j}$ is the state space of $\xi_{j}$, and $\mu_{\xi_{j}}$ its distribution. In the latter equality, we used the independence of $\xi_{j-1}(t N)<\xi_{j}(t N)$ and $\xi_{j}(t N)<\xi_{n}(t N)$ conditioned on $\xi_{j}=\pi$.

We claim now that $\mathbb{P}\left(\xi_{j-1}(t N)<\xi_{j}(t N) \mid \xi_{j}=\pi\right)$ decreases in $\pi$ and $\mathbb{P}\left(\xi_{j}(t N)<\xi_{n}(t N) \mid \xi_{j}=\pi\right)$ increases in $\pi$. (The reader should check it.) This and the FKG Inequality for $\mu_{\xi_{j}}$ (Lemma 2.6) imply that the right-hand side of (2.14) is bounded above by

$$
\sum_{j=2}^{n-1} \mathbb{P}\left(\xi_{j-1}(t N)<\xi_{j}(t N)\right) \mathbb{P}\left(\xi_{j}(t N)<\xi_{n}(t N)\right) \leqslant \mathbb{P}\left(\xi_{0}(t N)<\xi_{n}(t N)\right) \sum_{j=2}^{n-1} \mathbb{P}\left(\xi_{j-1}(t N)<\xi_{j}(t N)\right)
$$

since $\mathbb{P}\left(\xi_{j}(t N)<\xi_{n}(t N)\right)$ is clearly nonincreasing in $j$. Now the probabilities inside the sum are all bounded above by $\mathbb{P}\left(\xi^{(0,0)}(t N)<\xi^{\left(2 r_{0}, 0\right)}(t N)\right)$, and we get

$$
\begin{aligned}
\mathbb{P}\left(\eta^{\prime} \geqslant 3\right) & \leqslant n \mathbb{P}\left(\xi^{(0,0)}(t N)<\xi^{\left(2 r_{0}, 0\right)}(t N)\right) \mathbb{P}\left(\xi^{(0,0)}(t N)<\xi^{(\epsilon \sqrt{N}, 0)}(t N)\right) \\
& \leqslant \frac{\epsilon \sqrt{N}}{r_{0}} \mathbb{P}(\mathcal{T}>t N) \mathbb{P}\left(\mathcal{T}_{\epsilon, N}>t N\right),
\end{aligned}
$$

where $\mathcal{T}$ is the time when $\xi^{(0,0)}$ and $\xi^{\left(2 r_{0}, 0\right)}$ meet and coalesce, and $\mathcal{T}_{\epsilon, N}$ is the analogue time for $\xi^{(0,0)}$ and $\xi^{(\epsilon \sqrt{N}, 0)}$. 
Now, let us consider the items at the right-hand side of the latter equation. By Lemma 2.3,

$$
\limsup _{N \rightarrow \infty} \mathbb{P}\left(\mathcal{T}_{\epsilon, N}>t N\right)=\mathbb{P}\left(\mathcal{T}_{\epsilon, B}>t\right),
$$

where $\mathcal{T}_{\epsilon, B}$ is the time when two i.i.d. Brownian motions starting at the same time at distance $\epsilon$ apart meet and coalesce. Thus the latter probability is an $\mathrm{O}(\epsilon)$ for every $t>0$ fixed. By Lemma $2.7, \mathbb{P}(\mathcal{T}>t N) \leqslant c / \sqrt{t N}$. These estimates imply that

$$
\limsup _{N \rightarrow \infty} \mathbb{P}\left(\bar{\eta}_{X_{1}}(0, t N ; 0, \epsilon \sqrt{N}) \geqslant 3\right) \leqslant \limsup _{N \rightarrow \infty} \frac{\epsilon \sqrt{N}}{r_{0}} \mathbb{P}(\mathcal{T}>t N) \mathbb{P}\left(\mathcal{T}_{\epsilon, N}>t N\right)=\mathrm{O}\left(\epsilon^{2}\right),
$$

and we get $\mathrm{B}_{2}^{\prime}$ for $X_{\delta}$.

Remark. The argument for $\mathrm{B}_{2}$ in [7] for establishing weak convergence of coalescing random walks to the Brownian web also relies on an FKG property of the path distributions. But in that case, it is a stronger FKG property than in the present case. It allows bounding $\mathbb{P}(\eta \geqslant 3)$ above by $[\mathbb{P}(\eta \geqslant 2)]^{2}$, and then the use of $\mathrm{B}_{1}$. In particular, it is not necessary in that case to have an estimate of a microscopic quantity like $\mathbb{P}(\mathcal{T}>t N)$, on which we had to rely in here.

\section{Acknowledgements}

This work was begun when one of us (X.-Y. Wu) was visiting the Statistics Department of the Institute of Mathematics and Statistics of the University of São Paulo. He is thankful to the probability group of IME-USP for hospitality. We much thank Rongfeng Sun for pointing out important incorrections on Subsection 2.2 of an earlier version, as well as for subsequent discussions on our fixing of them.

\section{References}

[1] R. Arratia, Limiting point processes for rescalings of coalescing and annihilating random walks on $\mathbb{Z}^{d}$, Ann. Probab. 9 (1981) 909-936.

[2] M.D. Donsker, An invariance principle for certain probability limit theorems, Mem. Amer. Math. Soc. 6 (1951) 1-12.

[3] P.A. Ferrari, L.R.G. Fontes, X.-Y. Wu, Two-dimensional Poisson Trees converge to the Brownian web, math.PR/0304247.

[4] P.A. Ferrari, C. Landim, H. Thorisson, Poisson trees, succession lines and coalescing random walks, Ann. Inst. H. Poincaré 40 (2004) $141-152$.

[5] L.R.G. Fontes, M. Isopi, C.M. Newman, Random walks with strongly inhomogeneous rates and singular diffusions: convergence, localization and aging in one dimension, Ann. Probab. 30 (2002) 579-604.

[6] L.R.G. Fontes, M. Isopi, C.M. Newman, K. Ravishankar, The Brownian web, Proc. Nat. Acad. Sci. USA 99 (25) (2002) $15888-15893$.

[7] L.R.G. Fontes, M. Isopi, C.M. Newman, K. Ravishankar, The Brownian web: characterization and convergence, Ann. Probab. 32 (2004) 2857-2883.

[8] L.R.G. Fontes, M. Isopi, C.M. Newman, D.L. Stein, Aging in 1D discrete spin models and equivalent systems, Phys. Rev. Lett. 87 (2001) 110201.

[9] S. Gangopadhyay, R. Roy, A. Sarkar, Random oriented trees: a model of drainage networks, Ann. Appl. Probab. 14 (2004) $1242-1266$.

[10] O. Kallenberg, Foundations of Modern Probability, Springer-Verlag, 1997.

[11] H.B. Mann, A. Wald, On stochastic limit and order relations, Ann. Math. Statist. 14 (1943) 217-226.

[12] Y.V. Prohorov, Convergence of random processes and limits theorems in probability theory, Theory Probab. Appl. 1 (1956) 157-214.

[13] I. Rodriguez-Iturbe, A. Rinaldo, Fractal River Basins: Chance and Self-Organization, Cambridge University Press, New York, 1997.

[14] A.E. Scheidegger, A stochastic model for drainage patterns into an intramontane trench, Bull. Ass. Sci. Hydrol. 12 (1967) 15-20. 